\title{
Expression of Toll-Like Receptors in Neonatal Sepsis
}

\author{
DOROTHEE VIEMANN, GABRIELE DUBBEL, SUSANNE SCHLEIFENBAUM, ERIK HARMS, \\ CLEMENS SORG, AND JOHANNES ROTH
}

Department of Pediatrics [D.V., G.D., S.S., E.H., J.R.], Department of Experimental Dermatology

[D.V., C.S., J.R.], University of Münster, 48149 Münster, Germany

\section{ABSTRACT}

\begin{abstract}
Despite recent identification of specific pattern recognition receptors (PRR) for distinct microbial structures, data indicating their relevance in human infectious diseases are limited. We determined the expression levels of the Toll-like receptor (TLR) 2 and TLR4 by flow cytometry on granulocytes and monocytes of healthy neonates compared with healthy adults. The basal expression of TLR2 was only slightly lower in neonatal phagocytes, whereas no differences could be detected for TLR4. Analyzing neonates with sepsis, we found an impressive up-regulation of TLR2 on blood phagocytes already at initial presentation of symptoms. Comparison with C-reactive protein, IL- 8 , and IL- 6 suggested that TLR2 expression on monocytes is comparably valuable as an early sepsis marker. TLR2 was differentially regulated during neonatal sepsis, showing a constant up-regulation on monocytes but only a transient increase on
\end{abstract}

granulocytes. Surprisingly, TLR4 showed no remarkable changes. Our results revealed a mild deficiency of TLR2 expression in newborns and demonstrated a differential expression of TLR2 but not TLR4 in the course of neonatal sepsis, which could reflect specific inflammatory responses to distinct pathogens. The definition of TLR expression patterns might open a new field of therapeutic targets for neonatal sepsis. (Pediatr Res 58: 654-659, 2005)
LPS, lipopolysaccharide

\section{Abbreviations}
MFI, mean fluorescence intensity
PRR, pattern recognition receptor
TLR, Toll-like receptor

Neonatal sepsis remains a leading cause of neonatal morbidity and mortality despite recent advances in neonatal intensive care $(1,2)$. Although several deficiencies in the neonatal immune system have been revealed, including qualitative and quantitative deficits in phagocytes and humoral components (3-8), they may only partly explain the overwhelming nature of neonatal sepsis. The key for differences in the course of neonatal and adult sepsis might be molecular mechanisms involved in the initiation process of systemic inflammatory response syndromes, i.e. the activation of innate immune cells through invading microbes. In the innate immune system, many types of receptors participate in microbe detection. An exciting discovery was the finding that host organisms have developed a set of specific receptors (PRR) for the recognition of highly conserved and essential molecular structures of microbes [pathogen-associated molecular patterns, PAMP)] (9). TLR2 was identified as a receptor for Gram-positive peptidoglycan and bacterial lipopeptides $(10,11)$, whereas TLR4 is part of a receptor complex recognizing Gram-negative bacte-

Received February 8, 2005; accepted March 9, 2005.

Correspondence: Dorothee Viemann, M.D., Department of Pediatrics, University Hospital Münster, Albert-Schweitzer-Str. 33, 48149 Münster, Germany; e-mail: viemannd@ uni-muenster.de

DOI: 10.1203/01.PDR.0000180544.02537.FD rial LPS and is required for LPS signal transduction (12). Both finally trigger inflammatory responses through an IL-1 receptor-like pathway that uses an adapter protein (MyD88), IL-1 receptor associated kinase (IRAK), and tumor necrosis factor receptor-associated factor (TRAF)-6 signaling to activate nuclear factot $(\mathrm{NF}) \kappa \mathrm{B}$ and mitogen-activated protein kinase dependent signaling pathways (13). They initiate distinct genetic programs, including the induction of a subset of chemokines like macrophage inflammatory protein (MIP)- $1 \alpha$, MIP- $1 \beta$, and RANTES (regulated upon activation, normal t-cell expressed and secreted) mediated by TLR2 and TLR4, whereas IP-10 is preferentially induced by TLR4 and IL- 8 preferentially by TLR2 (14). The model of microbial recognition has now evolved into one in which immune cells use multiple PRR to detect several features of a microbe simultaneously. The signal pattern transduced by the combination of different innate immune receptors may be the key to the kind of immune response that is elicited (15).

Except for in vitro experiments or murine models, data regarding the expression and in vivo relevance of TLR in humans are limited. Analysis of TLR1, 2, and 4 in experimental endotoxemia indicated a differential regulation of the expression of TLR1, 2, and 4 on granulocytes and monocytes by LPS (16). A recent study in adults revealed up-regulation of 
TLR2 expression in Gram-negative and -positive sepsis without changes in the expression of TLR4 (17). We determined the expression levels of TLR2 and TLR4 on granulocytes and monocytes in healthy newborns and adults to obtain first information whether basal differences might explain the different courses of sepsis. Furthermore, we analyzed the expression of TLR2 and TLR4 in the course of neonatal sepsis to evaluate them as potential early sepsis markers compared with classical sepsis markers as CRP, IL-8, and IL-6 (18-20) and to asses the relevance of TLR in human infectious disease in general inasmuch as neonatal sepsis is more homogeneous than adult sepsis with respect to underlying causes.

\section{METHODS}

Study population. In this study, we prospectively enrolled 20 adult healthy blood donors and 85 term neonates without congenital malformation. Sepsis in newborns was defined according to generally accepted criteria as described earlier (21). In addition to clinical symptoms (apnea, bradycardia, desaturation, instability of body temperature, feeding intolerance), the presence of at least three of the following criteria within $48 \mathrm{~h}$ after onset of symptoms was required: a) C-reactive protein $(\mathrm{CRP})>20 \mathrm{mg} / \mathrm{L}$; b) pneumonia (radiologic diagnosis or cultural evidence in tracheal aspirate); c) cultural evidence of infection other than blood culture; d) ratio of immature to total neutrophils (I/T ratio) $>0.2$; and e) green amniotic fluid, premature rupture of membranes, or signs of infection of the mother. In addition, sepsis was proven if microbes were isolated from blood cultures. No death occurred; all patients could be successfully treated by supportive and standard antibiotic treatment. Control blood samples were obtained from healthy neonates in whom blood was taken for the screening of inborn errors of metabolism. The parents' informed consent was obtained and the study was approved by the institutional ethics committee.

Flow cytometry. EDTA whole blood samples were stained for $20 \mathrm{~min}$ at $4^{\circ} \mathrm{C}$ in the dark with isotype-matched control antibodies (BD PharMingen, San Diego, CA), a FITC-conjugated CD14 MAb (MAb) (clone M $\varphi$ P9, BD PharMingen), and PE-conjugated MAb against TLR2 (clone TL2.1, eBioscience, San Diego, CA) or TLR4 (clone HTA125, eBioscience), respectively. Measurements were performed using a FACSCalibur flow cytometer and data acquired by CellQuest software (BD Biosciences, Franklin Lakes, NJ). Granulocytes and monocytes were differentiated by scatter properties and CD14 $4^{\text {high }}$ (monocytes) or CD14 ${ }^{\text {low }}$ - (granulocytes) expression. For each marker, the MFI of the marker positive cell population was determined.

Data analysis. We defined the MFI shift as the ratio of the MFI of the marker to the MFI of the isotype control. For statistical analyses we applied the Mann-Whitney test and considered $p$ values $<0.05$ significant. Correlations of parameter were calculated according to the method of Spearman-Rho using the SPSS software version 11.0.1 (SPSS Inc., Chicago, IL).

Analysis of $C R P, I L-8$, and IL-6 serum levels. CRP serum concentrations were determined by a commercial chemoluminescence-immunoassay (IMMULITE, DPC Biermann, Bad Nauheim, Germany). The concentrations of IL-8 and IL-6 were determined using the human inflammation cytometric bead array (CBA) (BD PharMingen). Briefly, $10 \mu \mathrm{L}$ of each serum sample was mixed with $50 \mu \mathrm{L}$ of mixed capture beads and incubated for $1.5 \mathrm{~h}$ at room temperature. Samples were washed once and incubated for $1.5 \mathrm{~h}$ at room temperature in the dark with $50 \mu \mathrm{L}$ of the human inflammation PE detection reagent containing PE-conjugated anti-human IL-8 and anti-human IL-6. Subsequently, samples were washed and resuspended in $300 \mu \mathrm{L}$ wash buffer before acquisition on the FACSCalibur using CBA software (BD PharMingen). Standard curves were generated using the standards provided by the kit. The concentrations for IL-8 and IL- 6 were determined by interpolation from the standard curves ranging from 20 to $5000 \mathrm{pg} / \mathrm{mL}$, respectively.

\section{RESULTS}

Neonatal study population. We restricted the study population to term neonates without congenital malformations. Healthy newborns $(n=53)$ and patients $(n=32)$ were prospectively enrolled and matched for gender, gestational age, and birth weight (Table 1). In the majority of cases, first symptoms of sepsis were seen at the first or second day of life (early-onset sepsis, $n=27$ ), whereas sepsis after $72 \mathrm{~h}$ of birth occurred in only five cases. Control samples were matched according to the age at entry in the study (Table 1). In six patients, sepsis was proven by positive blood culture, from which Gram-positive microbes were isolated in five cases (group B streptococci) and Gram-negative in one case (Escherichia coli).

Basal differences of TLR expression in healthy newborns and adults. To determine whether basal differences in the expression pattern of TLR exist between healthy neonates $(n=$ 53) and adults $(n=20)$, we analyzed the expression of TLR2 and TLR4 on phagocytic cells by flow cytometry. Except for the expression of TLR2 on adult monocytes, the expression level of TLR2 and TLR4 were low in phagocytic cells of healthy individuals (Fig. 1). TLR2 was expressed at somewhat higher levels in adult than in newborn phagocytes (1.2-fold in granulocytes, 1.6-fold in monocytes) (Fig. 1A). The expression level of TLR4 showed a higher variance in neonatal granulocytes and monocytes and no significant differences between adults and neonates (Fig. 1B).

Differential expression of TLR on granulocytes and monocytes in the course of neonatal sepsis. We performed flow cytometric analysis of TLR2 and TLR4 expression in newborn patients on $\mathrm{d} 0$, when clinical sepsis was first suspected and before start of antibiotic treatment, and at four defined time points during follow-up (d 1, 2/3, 4-6, and 7-10). Distinct scatter properties as well as expression levels of CD14 allowed the flow cytometric differentiation of granulocytes and monocytes and isolated analysis of TLR2 and TLR4 (Fig. 2, $A$ and $B$ ).

Already at initial presentation patients with sepsis showed a significant increase of TLR2 expression on granulocytes and monocytes compared with healthy neonates $(p<0.01)$ with a general higher expression level in monocytes (Fig. 2, $A$ and $B$, and Fig. 3, $A$ and $B$ ). Taking the mean value plus $2 \mathrm{SD}$ of the control group as an upper cut-off, we found an elevated expression of TLR2 on d 0 of sepsis in $24 \%$ of all patients for granulocytes and in $71 \%$ of all patients for monocytes. In granulocytes, TLR2 expression further increased on d 1 of sepsis up to $63 \%$ of patients but then rapidly decreased to control values until the end of the first week to insignificant values in comparison to the control. In contrast, in monocytes, the expression of TLR2 remained constantly high until the second week of sepsis. The expression changes of TLR2 were even stronger in patients with bacteremia as documented by positive blood cultures (MFI ratio \pm SEM in granulocytes: $\mathrm{d} 0$, $1,2 / 3,4-6$, and $7-10$ of unproven sepsis: $2.13 \pm 0.20,3.24 \pm$ $0.68,2.33 \pm 0.22,1.51 \pm 0.22$, and $1.77 \pm 0.20 ; \mathrm{d} 0,1$, $2 / 3,4-6$, and $7-10$ of proven sepsis: $2.59 \pm 0.34,3.3 \pm 0.41$,

Table 1. Characteristics of study groups

\begin{tabular}{lcc}
\hline & $\begin{array}{c}\text { Sepsis group } \\
(n=32)\end{array}$ & $\begin{array}{c}\text { Control group } \\
(n=53)\end{array}$ \\
\hline Sex (F/M) & $10 / 22$ & $25 / 28$ \\
Birthweight (g) & $3500 \pm 420$ & $3200 \pm 690$ \\
Gestational age (weeks) & $39 \pm 2$ & $39 \pm 3$ \\
APGAR 1 min & $7 \pm 2$ & $9 \pm 1$ \\
APGAR 5 min & $9 \pm 1$ & $10 \pm 1$ \\
Age at entry in the study (days) & $2 \pm 2$ & $3 \pm 2$ \\
\hline
\end{tabular}


A.

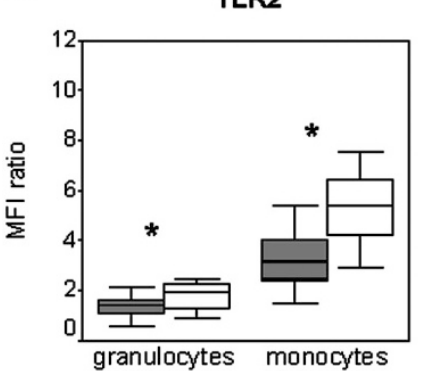

B.

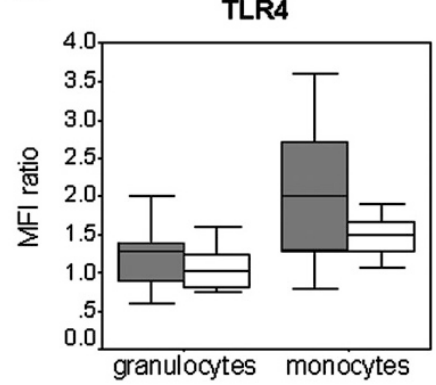

A.

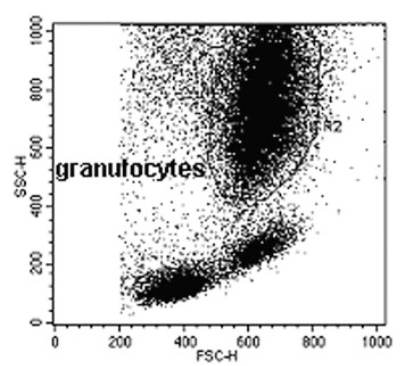

C. blood samples from healthy newborns (dark boxplots, $n=53$ ) and healthy adults (light boxplots, $n=20$ ) were obtained. Analyses of the expression of TLR2 $(A)$ and TLR4 $(B)$ were performed by flow cytometry, allowing the differentiation of granulocytes and monocytes by scatter properties. Boxplots represent ranges, quartiles, and medians. *Significant differences between neonatal and adult cells, with $p<0.01$.

$3.65 \pm 0.82,2.25 \pm 0.43$, and $1.91 \pm 0.42$. MFI ratio \pm SEM in monocytes: $\mathrm{d} 0,1,2 / 3,4-6$, and $7-10$ of unproven sepsis: $1.47 \pm 0.20,1.80 \pm 0.24,2.24 \pm 0.33,1.71 \pm 0.19$, and $2.25 \pm 0.24 ; \mathrm{d} 0,1,2 / 3,4-6$, and $7-10$ of proven sepsis: $2.12 \pm 0.27,1.67 \pm 0.18,2.76 \pm 0.34,2.48 \pm 0.46$, and $3.00 \pm 0.30$ ).

The expression of TLR4 on granulocytes remained constantly low and showed no significant changes (Fig. $2 A$ and Fig. 3A). On monocytes, the expression level of TLR4 was higher compared with granulocytes and increased slightly during sepsis but was significant only on d 1 compared with control monocytes (Fig. $2 B$ and Fig. $3 B$ ) d 2 or 3.

Correlation of TLR expression with WBC counts. To control whether changes in the expression of TLR2 on granulocytes and monocytes might be due to shifts of absolute cell counts or shifts of granulocytes to bands in the course of neonatal sepsis, we performed blood counts according to the time schedule applied for the flow cytometric analyses, i.e. $\mathrm{d} 0$, $1,2 / 3,4-6$, and 7-10 of sepsis. Monocyte counts did not change in the course of sepsis (Table 2), suggesting that expression changes of TLR2 detected on monocytes are rather due to activation and maturation processes than a release of new subpopulations of monocytes from the bone marrow. As anticipated, significant increases on d 0 of sepsis could be detected for the number of leukocytes, bands, and granulocytes (Table 2), implying a relation to the changes in the expression of TLR2 on granulocytes. However, with a correlation coefficient $r$ of $0.18(p>0.1)$, no significant relation exists between the expression levels of TLR2 and the course of leukocyte or band counts during neonatal sepsis. Only with respect to the number of granulocytes did we find a similar time course compared with the expression of TLR2. However, the correlation was poor, with $r=0.29$ ( $p<0.005)$.

Value of the analysis of TLR expression levels as early sepsis marker. The high expression of TLR2 on phagocytic cells upon presentation of neonatal sepsis patients suggests this parameter as potential early sepsis marker. We, therefore, randomly selected 12 control newborns and 12 sepsis patients to analyze CRP, IL-8, and IL- 6 on d 0 of sepsis. We compared

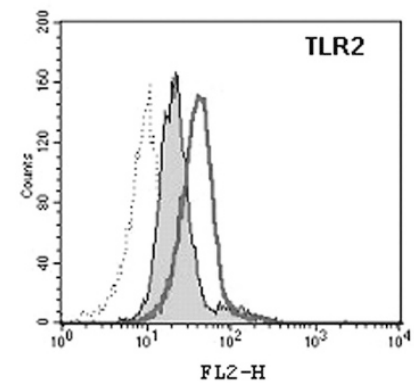

E.

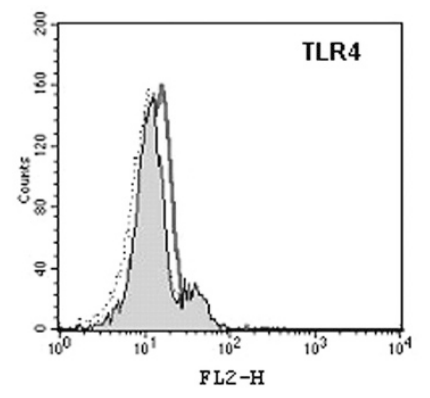
neonatal sepsis. tive) (Fig. 4).
B.

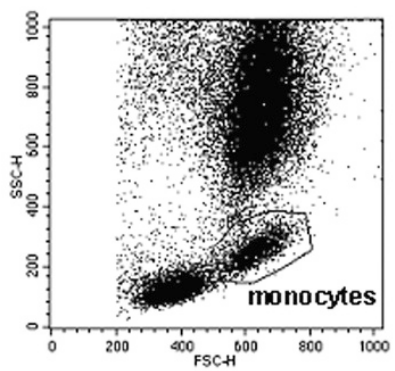

D.

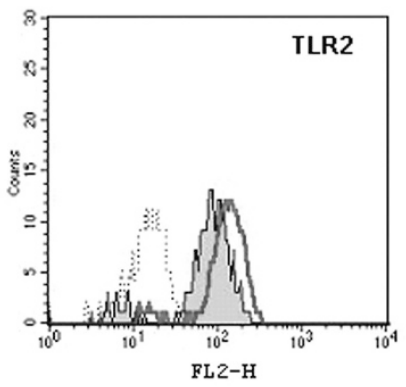

F.

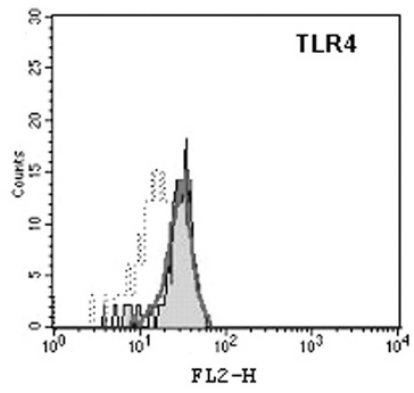

Figure 2. TLR2 and TLR4 expression pattern at initial presentation of neonatal sepsis. Granulocytes $(A, C, E)$ and monocytes $(B, D, F)$ were differentiated flow cytometrically by scatter properties. We determined the expression of TLR2 $(C, D)$ and TLR4 $(E, F)$ according to the appropriate PE-conjugated isotype matched control antibody (dotted line) in healthy neonates (gray-filled area) and neonates on d 0 of sepsis (bold line). Histograms show representative data from one healthy newborn and one patient with

the serum level of these inflammation markers with the corresponding expression levels of TLR2 in this study group.

With respect to the upper cut-off (mean value +2 SD of the control group), we found elevated CRP serum concentrations in 8 patients $(67 \%)$ (data not shown), elevated IL-8 serum levels in 7 (58\%), and elevated IL-6 levels in 8 of 12 patients $(67 \%)$ on $\mathrm{d} 0$ of sepsis (Fig. 4A). In the 12 healthy newborns, CRP (data not shown) and IL-8 (Fig. 4) were not increased (no false positive) and IL-6 was only once increased ( $8 \%$ false positive) (Fig. 4A). The same approach applied on TLR2 expression revealed elevated levels on monocytes in 8 of 12 patients $(67 \%)$ on $\mathrm{d} 0$ of sepsis and 1 of 12 control newborns comparable to the sensitivity and specificity of IL-6 serum levels (Fig. 4). TLR2 expression on granulocytes was not a competitive sepsis marker (33\% true positive, $8 \%$ false posi- 

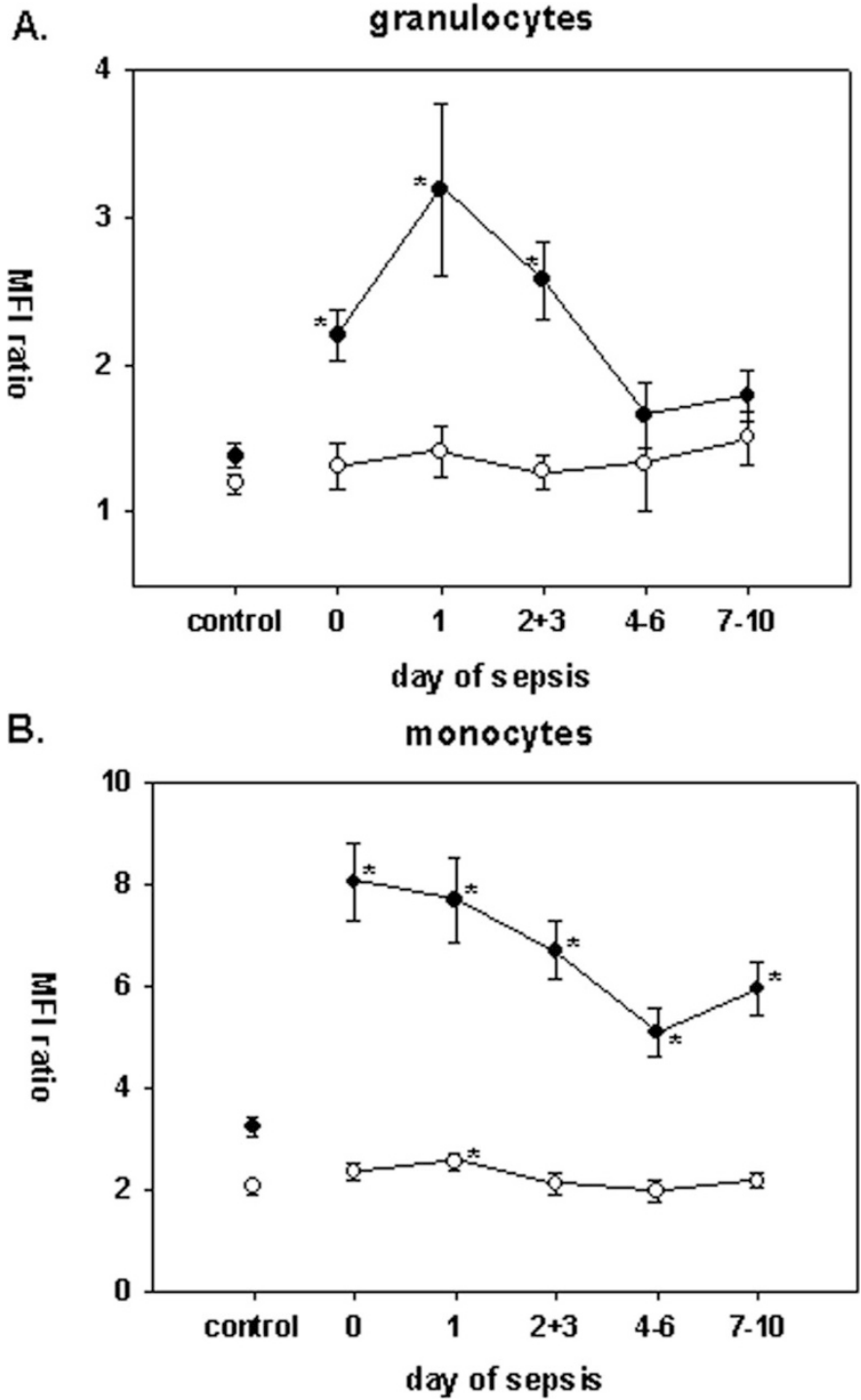

Figure 3. Expression of TLR 2 and TLR 4 in the course of neonatal sepsis. The expression level of TLR2 $(\bullet)$ and TLR4 $(\bigcirc)$ were analyzed by flow cytometry on granulocytes $(A)$ and monocytes $(B)$ and expressed as MFI ratio. Data were acquired from healthy newborns (controls, $n=53)$ and patients $(n=32)$ on four defined time points during the course of neonatal sepsis. Plots represent means with SEM. *Significant differences in the expression levels of control group and sepsis group, with $p<0.01$.

\section{DISCUSSION}

Neonatal sepsis causes significant mortality and morbidity in infants, especially preterm infants $(1,2,22)$. Despite considerable advances of neonatal intensive care medicine, the patho- genesis of neonatal sepsis with its rapid progression from infection to a systemic inflammatory response still remains little understood. Beyond doubt, sepsis is a clinical manifestation of a dysregulated immune response to invasive pathogens. However, numerous clinical trials with immunomodulatory approaches for neonates with suspected or proven infections failed to improve survival (23-26). Many new concepts regarding the pathogenesis of sepsis evolved from the recent finding of a diverse system of PRR based on in vitro experiments or data obtained in the murine system (15). Thus, mice with a genetic deficiency or mutations of TLR4 are defective in LPS signaling and more resistant to endotoxin shock (12), whereas TLR2- and MyD88-deficient mice are more susceptible to infections by Staphylococcus aureus (27). Major evidence of a pathophysiological relevance of TLR in infectious diseases derives from a recent study in a murine model of experimental polymicrobial sepsis. TLR2 and TLR4 gene expression and TLR4 protein levels increased in liver and lung $24 \mathrm{~h}$ after induction of sepsis, which correlated strongly with sepsisinduced lethality (28). The first in vivo study in humans with respect to the expression of TLR was conducted in experimental endotoxemia in adults showing up-regulation of TLR2 on monocytes but no changes on neutrophils after LPS infusion, whereas TLR4 was strongly down-regulated on neutrophils but not significantly regulated in monocytes (16). A recent study in adults with proven sepsis investigated the expression of TLR2 and TLR4 only on monocytes, confirming the results of experimental endotoxemia (17).

Data for healthy neonates with respect to the expression of TLR at the protein level are limited $(29,30)$ and no data have been reported so far for neonates with infections. Our study reveals several differences between neonates and adults regarding the expression of TLR2 and TLR4. In healthy individuals, TLR2 is significantly but rather little lower expressed on neonatal granulocytes and monocytes compared with adult phagocytes, whereas the expression levels of TLR4 are comparable. A differential maturation deficiency regarding the expression of TLR in neonates becomes obvious. In recent studies, the basal expression of TLR2 $(29,30)$ and TLR4 $(29,30)$ were similar in neonatal and adult monocytes at the mRNA and/or protein level. However, these studies were performed using umbilical cord blood. Our analysis of peripheral blood samples confirmed no differences for TLR4 but revealed little while significant lower basal TLR2 expression on neonatal phagocytes. Interestingly, the characterization of TLR2-deficient mice revealed that local infection with group B streptococci (GBS) lead to systemic spread of GBS, which

Table 2. Absolute values of leucocytes

\begin{tabular}{lcccc}
\hline & $\begin{array}{c}\text { Leucocytes* } \\
\left(\times 10^{3} / \mu 1\right)\end{array}$ & $\begin{array}{c}\text { Bands* } \\
(/ \mu 1)\end{array}$ & $\begin{array}{c}\text { Granulocytes* } \\
\left(\times 10^{3} / \mu 1\right)\end{array}$ & $\begin{array}{c}\text { Monocytes* } \\
\left(\times 10^{3} / \mu 1\right)\end{array}$ \\
\hline Control group & $12.8 \pm 6.2$ & $114.9 \pm 74.6$ & $7.4 \pm 4.9$ & $1.6 \pm 3.8$ \\
Sepsis group/day 0 & $19.5 \pm 7.7 * *$ & $1231.3 \pm 444.0^{* *}$ & $12.2 \pm 6.7^{* *}$ & $1.0 \pm 0.7$ \\
Sepsis group/day 1 & $16.1 \pm 10.7$ & $626.4 \pm 536.0^{* *}$ & $10.0 \pm 9.1$ & $1.0 \pm 0.8$ \\
Sepsis group/day 2 +3 & $13.4 \pm 7.3$ & $213.8 \pm 211.7$ & $7.9 \pm 5.9$ & $1.0 \pm 0.7$ \\
Sepsis group/day 4-6 & $13.3 \pm 4.3$ & $133.0 \pm 55.4$ & $6.0 \pm 3.4$ & $1.5 \pm 1.0$ \\
Sepsis group/day 7-10 & $11.3 \pm 2.2$ & $101.6 \pm 24.1$ & $3.9 \pm 1.4^{* *}$ & $1.3 \pm 0.6$ \\
\hline
\end{tabular}

$*$ Mean values $\pm \mathrm{SD}$. ** Differences significant with $p$-values $<0.05$ compared to the control group. 


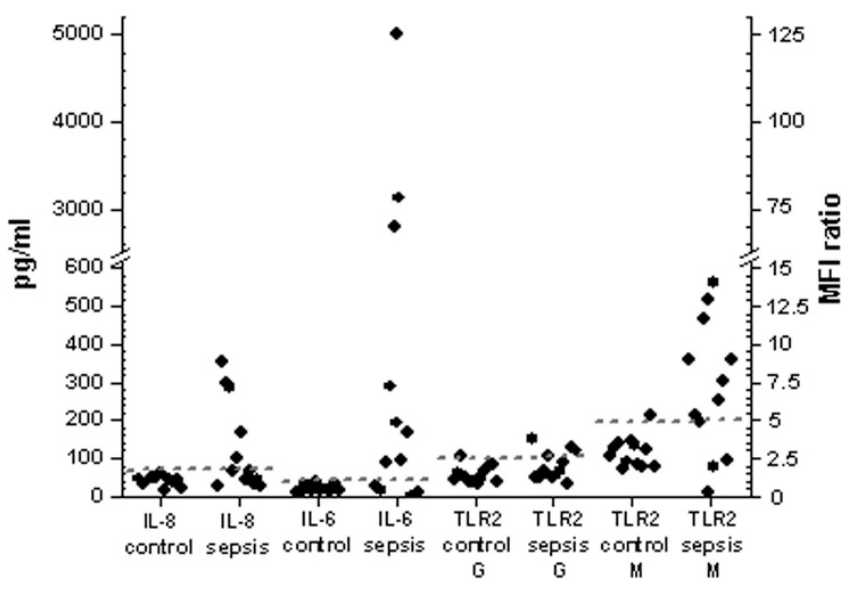

Figure 4. TLR 2 expression level as early sepsis parameter. IL-8 and IL-6 serum concentrations plotted on the left $y$ axis were determined in control newborns $(n=12)$ and patients on $\mathrm{d} 0$ of sepsis $(n=12)$. Expression levels of TLR2 were analyzed on granulocytes $(G)$ and monocytes $(M)$ and plotted on the right $y$ axis as MFI ratio. The cut-offs (dotted lines) were defined as mean values $+2 \mathrm{SD}$ of the control group.

could be prevented in wild-type mice (31). Our results of a low basal expression of TLR2 might therefore be associated with the particular susceptibility of neonates for infections with Gram-positive pathogens.

Our data show that the expression of TLR2 on phagocytes is already increased at initial presentation of clinical symptoms. The majority of patients show values above the $95 \%$ confidence interval for the expression of TLR2 on monocytes (67\%). From that point of view, TLR2 might compete with classic sepsis markers as CRP, IL-8, and IL-6, for which broad sensitivity ranges of $28-89 \%$ for CRP, $50-92 \%$ for IL-8, and $74-100 \%$ for IL-6 were reported (18,32-34). In our study, the direct comparison of CRP, IL-8, and IL-6 with TLR expression in a group 24 healthy and septic newborns showed that TLR2 expression on monocytes appeared similarly valuable.

Moreover, TLR2 was differentially regulated in the course of neonatal sepsis, with a rapid and constant high expression on monocytes but only transient up-regulation on granulocytes. With respect to the expression pattern on monocytes, data are concordant with analyses in adult experimental endotoxemia and adult sepsis. However, adults showed no changes in the expression of TLR2 on granulocytes, supporting the hypothesis of a hyperinflammatory state in neonatal sepsis $(16,17)$. Data analysis showed a weak correlation between the course of TLR2 expression and the course of granulocyte counts, implying activation or maturation processes of the granulocytes leading to a specific loss of TLR2, for example, through shedding or internalization. However, a simple consumption of granulocytes leading to the decrease in TLR2 expression cannot be excluded. The down-regulation of the TLR2-mediated inflammatory program in granulocytes after $3 \mathrm{~d}$ of sepsis also potentially reflects successful treatment. The constant high TLR2 expression on monocytes suggests a predominant role for monocytes in the later phase of sepsis. In vitro assays have demonstrated that effector activity and survival of granulocytes in response to LPS depend upon the presence of monocytes (35). Therefore, in neonatal sepsis, ongoing inflammatory ac- tivity of monocytes might be mandatory for a successful outcome.

Surprisingly, TLR4 showed no remarkable expression changes in the course of neonatal sepsis. Because TLR4 primarily recognizes LPS of Gram-negative bacteria (12) and expression levels correlate with LPS susceptibility in mice (36), the entire expression pattern in neonatal sepsis might reflect the fact that Gram-positive microorganisms are responsible for the majority of cases. In experimental endotoxemia and adults with Gram-positive as well as Gram-negative sepsis, TLR4 expression on monocytes did not change and decreased on granulocytes in experimental endotoxemia $(16,17)$.

In summary, we demonstrate for the first time expression deficits of TLR2 on neonatal innate immune cells compared with adults. Furthermore, we observed distinct expression patterns of TLR2 and TLR4 in the course of neonatal sepsis. Rapid and high increase of the expression of TLR2 at initial symptoms suggests this parameter as a candidate for early sepsis marker. Because therapeutic modulation of TLR promised high benefit for sepsis outcome in a murine model (28), the definition of TLR expression patterns might open a new field of therapeutic targets for neonatal sepsis.

\section{REFERENCES}

1. Stoll BJ, Gordon T, Korones SB, Shankaran S, Tyson JE, Bauer CR, Fanaroff AA, Lemons JA, Donovan EF, Oh W, Stevenson DK, Ehrenkranz RA, Papile LA, Verter J, Wright LL 1996 Early-onset sepsis in very low birth weight neonates: a report from the National Institute of Child Health and Human Development Neonatal Research Network. J Pediatr 129:72-80

2. Stoll BJ, Gordon T, Korones SB, Shankaran S, Tyson JE, Bauer CR, Fanaroff AA, Lemons JA, Donovan EF, Oh W, Stevenson DK, Ehrenkranz RA, Papile LA, Verter J, Wright LL 1996 Late-onset sepsis in very low birth weight neonates: a report from the National Institute of Child Health and Human Development Neonatal Research Network. J Pediatr 129:63-71

3. Abughali N, Berger M, Tosi MF 1994 Deficient total cell content of CR3 (CD11b) in neonatal neutrophils. Blood 83:1086-1092

4. Andersson U, Bird AG, Britton BS, Palacios R 1981 Humoral and cellular immunity in humans studied at the cell level from birth to two years of age. Immunol Rev $57: 1-38$

5. Carr R 2000 Neutrophil production and function in newborn infants. Br J Haematol $110: 18-28$

6. Hill HR 1987 Biochemical, structural, and functional abnormalities of polymorphonuclear leukocytes in the neonate. Pediatr Res 22:375-382

7. Kampalath B, Cleveland RP, Kass L 1998 Reduced CD4 and HLA-DR expression in neonatal monocytes. Clin Immunol Immunopathol 87:93-100

8. Viemann D, Schlenke P, Hammers HJ, Kirchner H, Kruse A 2000 Differential expression of the B cell-restricted molecule CD22 on neonatal B lymphocytes depending upon antigen stimulation. Eur J Immunol 30:550-559

9. Medzhitov R, Janeway CA Jr 1997 Innate immunity: the virtues of a nonclonal system of recognition. Cell 91:295-298

10. Schwandner R, Dziarski R, Wesche H, Rothe M, Kirschning CJ 1999 Peptidoglycanand lipoteichoic acid-induced cell activation is mediated by toll-like receptor 2. J Biol Chem 274:17406-17409

11. Yoshimura A, Lien E, Ingalls RR, Tuomanen E, Dziarski R, Golenbock D 1999 Cutting edge: recognition of Gram-positive bacterial cell wall components by the innate immune system occurs via Toll-like receptor 2. J Immunol 163:1-5

12. Poltorak A, He X, Smirnova I, Liu MY, Van Huffel C, Du X, Birdwell D, Alejos E, Silva M, Galanos C, Freudenberg M, Ricciardi-Castagnoli P, Layton B, Beutler B 1998 Defective LPS signaling in $\mathrm{C} 3 \mathrm{H} / \mathrm{HeJ}$ and $\mathrm{C} 57 \mathrm{BL} / 10 \mathrm{ScCr}$ mice: mutations in Tlr4 gene. Science 282:2085-2088

13. Ulevitch RJ 2003 Regulation of receptor-dependent activation of the innate immune response. J Infect Dis 187(suppl 2):S351-S355

14. Luster AD 2002 The role of chemokines in linking innate and adaptive immunity. Curr Opin Immunol 14:129-135

15. Underhill DM, Ozinsky A 2002 Toll-like receptors: key mediators of microbe detection. Curr Opin Immunol 14:103-110

16. Marsik C, Mayr F, Cardona F, Derhaschnig U, Wagner OF, Jilma B 2003 Endotoxaemia modulates Toll-like receptors on leucocytes in humans. Br J Haematol 121:653-656

17. Armstrong L, Medford AR, Hunter KJ, Uppington KM, Millar AB 2004 Differential expression of Toll-like receptor (TLR)-2 and TLR-4 on monocytes in human sepsis. Clin Exp Immunol 136:312-319

18. Chiesa C, Pellegrini G, Panero A, Osborn JF, Signore F, Assumma M, Pacifico L 2003 C-reactive protein, interleukin-6, and procalcitonin in the immediate postnatal 
period: influence of illness severity, risk status, antenatal and perinatal complications, and infection. Clin Chem 49:60-68

19. Laborada G, Rego M, Jain A, Guliano M, Stavola J, Ballabh P, Krauss AN, Auld PA, Nesin M 2003 Diagnostic value of cytokines and C-reactive protein in the first 24 hours of neonatal sepsis. Am J Perinatol 20:491-501

20. Ng PC 2004 Diagnostic markers of infection in neonates. Arch Dis Child Fetal Neonatal Ed 89:F229-F235

21. Kuster H, Weiss M, Willeitner AE, Detlefsen S, Jeremias I, Zbojan J, Geiger R, Lipowsky G, Simbruner G 1998 Interleukin-1 receptor antagonist and interleukin-6 for early diagnosis of neonatal sepsis 2 days before clinical manifestation. Lance 352:1271-1277

22. Moore MR, Schrag SJ, Schuchat A 2003 Effects of intrapartum antimicrobia prophylaxis for prevention of group-B-streptococcal disease on the incidence and ecology of early-onset neonatal sepsis. Lancet Infect Dis 3:201-213

23. Carr R, Modi N, Dore C 2003 G-CSF and GM-CSF for treating or preventing neonatal infections. Cochrane Database Syst Rev CD003066

24. Mohan P, Brocklehurst P 2003 Granulocyte transfusions for neonates with confirmed or suspected sepsis and neutropaenia. Cochrane Database Syst Rev CD003956

25. Ohlsson A, Lacy JB 2004 Intravenous immunoglobulin for suspected or subsequently proven infection in neonates. Cochrane Database Syst Rev CD001239

26. Suri M, Harrison L, Van de Ven C, Cairo MS 2003 Immunotherapy in the prophylaxis and treatment of neonatal sepsis. Curr Opin Pediatr 15:155-160

27. Takeuchi O, Hoshino K, Akira S 2000 Cutting edge: TLR2-deficient and MyD88 deficient mice are highly susceptible to Staphylococcus aureus infection. J Immunol 165:5392-5396

28. Williams DL, Ha T, Li C, Kalbfleisch JH, Schweitzer J, Vogt W, Browder IW 2003 Modulation of tissue Toll-like receptor 2 and 4 during the early phases of polymicrobial sepsis correlates with mortality. Crit Care Med 31:1808-1818
29. Levy O, Zarember KA, Roy RM, Cywes C, Godowski PJ, Wessels MR 2004 Selective impairment of TLR-mediated innate immunity in human newborns: neonatal blood plasma reduces monocyte TNF-alpha induction by bacterial lipopeptides, lipopolysaccharide, and imiquimod, but preserves the response to R-848. J Immunol 173:4627-4634

30. Yan SR, Qing G, Byers DM, Stadnyk AW, Al Hertani W, Bortolussi R 2004 Role of MyD88 in diminished tumor necrosis factor alpha production by newborn mononuclear cells in response to lipopolysaccharide. Infect Immun 72:1223-1229

31. Mancuso G, Midiri A, Beninati C, Biondo C, Galbo R, Akira S, Henneke P, Golenbock D, Teti G 2004 Dual role of TLR2 and myeloid differentiation factor 88 in a mouse model of invasive group B streptococcal disease. J Immunol 172:63246329

32. Franz AR, Kron M, Pohlandt F, Steinbach G 1999 Comparison of procalcitonin with interleukin 8, C-reactive protein and differential white blood cell count for the early diagnosis of bacterial infections in newborn infants. Pediatr Infect Dis J 18:666-671

33. Martin H, Olander B, Norman M 2001 Reactive hyperemia and interleukin 6, interleukin 8 , and tumor necrosis factor-alpha in the diagnosis of early-onset neonatal sepsis. Pediatrics 108:E61

34. Santana C, Guindeo MC, Gonzalez G, Garcia-Munoz F, Saavedra P, Domenech E 2001 Cord blood levels of cytokines as predictors of early neonatal sepsis. Acta Paediatr 90:1176-1181

35. Sabroe I, Jones EC, Usher LR, Whyte MK, Dower SK 2002 Toll-like receptor (TLR)2 and TLR4 in human peripheral blood granulocytes: a critical role for monocytes in leukocyte lipopolysaccharide responses. J Immunol 168:4701-4710

36. Feterowski C, Emmanuilidis K, Miethke T, Gerauer K, Rump M, Ulm K, Holzmann B, Weighardt H 2003 Effects of functional Toll-like receptor-4 mutations on the immune response to human and experimental sepsis. Immunology 109:426-431 